\title{
Pain In Extremity, CTCAE
}

National Cancer Institute

\section{Source}

National Cancer Institute. Pain In Extremity, CT CAE. NCI Thesaurus. Code C143723.

A disorder characterized by a sensation of marked discomfort in the upper or lower extremities. 